Nervenarzt 2020 $91: 383-384$

https://doi.org/10.1007/s00115-020-00897-x

(c) Springer Medizin Verlag GmbH, ein Teil von Springer Nature 2020

\author{
A. Heinz $z^{1}$ K.-M. Perrar ${ }^{2} \cdot$ R. Voltz ${ }^{2}$ \\ ' Klinik für Psychiatrie und Psychotherapie CCM, Charité - Universitätsmedizin Berlin, Berlin, Deutschland \\ ${ }^{2}$ Zentrum für Palliativmedizin, Uniklinik Köln, Köln, Deutschland
}

\title{
Palliativmedizin und Psychiatrie - eine kontraintuitive Beziehung?
}

Palliativmedizin ist für Psychiatrie und Psychotherapie bedeutsamer als vielfach vermutet. Es gibt drei große Missverständnisse in Bezug auf den Begriff „Palliativmedizin“. Erstens wird er oft mit der Versorgung in der Sterbephase, also mit den letzten Stunden und Tagen assoziiert. Dagegen ist international das Konzept einer Integration der Palliativmedizin $z u$ einem früheren Zeitpunkt des Krankheitsverlaufes, z. B. dem parallelen Hinzukommen bereits 12 bis 24 Monate vor dem vermuteten Versterben, längst anerkannt.

Zweitens wird der Begriff - weil historisch so entstanden - oft ausschließlich für die Versorgung von Krebspatientinnen und -patienten mitgedacht. Andere nichtheilbare, progredient verlaufende Erkrankungen, wie z. B. neurologische, nephrologische, pulmologische, aber auch psychiatrische Erkrankungen scheinen da zunächst nicht zu passen; aber alle Regelungen unseres Gesundheitssystems zur Palliativversorgung sprechen eben nicht von einzelnen Krankheitsentitäten, sondern immer von lebenslimitierenden Überlebensprognosen und damit verbundenem Leid.

\section{》) Jeder, der mit schwerkranken Menschen zu tun hat, ist palliativmedizinisch tätig}

Und drittens wird der Begriff oft assoziiert mit der Notwendigkeit des Einbeziehens von spezialisierten Palliativmedizinern; richtig ist aber, dass jeder, der mit schwerkranken Menschen zu tun hat, sich auch palliativmedizinisch betätigt, nur in besonders komplexen Situationen sollte der spezialisierte Palliativdienst dazu- kommen. In diesem Sinne ist die Psychiatrie schon seit vielen Jahrzehnten auch eine „palliative“ Disziplin.

Gibt es eine sinnhafte Beziehung zwischen Psychiatrie und Palliativmedizin? Historisch betrachtet stand die Begründerin der modernen Hospiz- und Palliativmedizin, Dame Cicely Saunders, von Anfang an in enger Kooperation mit einem Psychiater, Colin Murray Parkes, der sich mit seinen Arbeiten zur Trauer international einen Namen gemacht hat. Cicely Saunders konzentrierte sich in ihrer Arbeit auf Krebspatientinnen und -patienten, da damals Opiate zur Schmerztherapie neu in die Medizin Eingang fanden und sie dort - wie sie selbst sagt sehr viel bewirken konnte. In ihrem Gesamtkonzept hatte sie jedoch bereits auch Platz für Patienteninnen und Patienten mit z. B. der neurologischen Erkrankung amyotrophe Lateralsklerose, war also von Anfang an offen für andere Patientengruppen.

Wie stellen sich heute die Beziehung von Palliativmedizin und Psychiatrie dar? Ausgehend von den betroffenen Patientengruppen kann man hier, wie es in einem Versorgungsbericht im Auftrag des Schweizer Bundesamtes für Gesundheit (BAG 2014) vorgeschlagen wurde, drei Patientengruppen unterscheiden:

1. somatisch schwer erkrankte Patientinnen und Patienten in einer palliativen Versorgungssituation, die psychiatrische Komorbiditäten wie z. B. Anpassungsstörungen, Ängste, Depression oder ein Delir entwickeln,

2. psychisch schwer kranke Patientinnen und Patienten, die aufgrund kurativ nicht behandelbarer somatischer Erkrankungen eine Palliativversorgung benötigen und schließlich 
3. - und das ist die wahrscheinlich am ehesten kontroverse Patientengruppe - gibt es Patientinnen und Patienten mit einer schwerwiegenden, weitestgehend "therapierefraktären“ psychischen Erkrankung und einer eingeschränkten Lebenserwartung, wie dies z. B. bei Anorexien oder schweren Abhängigkeitserkrankungen der Fall sein kann.

Bei allen drei Patientengruppen lohnt es sich hinzusehen, wie die Versorgung dieser Menschen in Zukunft weiter verbessert werden kann und welchen Beitrag diesbezüglich eine palliative Haltung leisten kann. Zum Teil eröffnen sich hier klinisch Möglichkeiten, zum Teil aber auch komplexere ethische und juristische Fragestellungen, die auch die jeweils eigene therapeutische Haltung und die ihr zugrunde liegenden Werte betreffen.

Dieses Leitthemenheft stellt eine erste Bestandsaufnahme der neu gegründeten „Taskforce“ der Deutschen Gesellschaft für Psychiatrie und Psychotherapie, Psychosomatik und Nervenheilkunde und der Deutschen Gesellschaft für Gerontopsychiatrie und -psychotherapie mit der Deutschen Gesellschaft für Palliativmedizin dar, um eine gemeinsame Diskussion zu beginnen. Natürlich gibt es weitere in diesem Heft nicht behandelte Themen und es zeigt sich in vielen Bereichen großer Forschungsbedarf. Wir freuen uns aber gemeinsam mit Ihnen auf eine gewinnbringende Diskussion, nicht nur in diesem Heft.

Prof. Dr. Andreas Heinz

Dr. Klaus-Michael Perrar

Prof. Dr. Raymond Voltz

\section{Korrespondenzadresse}

Prof. Dr. R. Voltz

Zentrum für Palliativmedizin, Uniklinik Köln 50924 Köln, Deutschland raymond.voltz@uk-koeln.de

Interessenkonflikt. A. Heinz, K.-M. Perrar und R. Voltz geben an, dass kein Interessenkonflikt besteht.
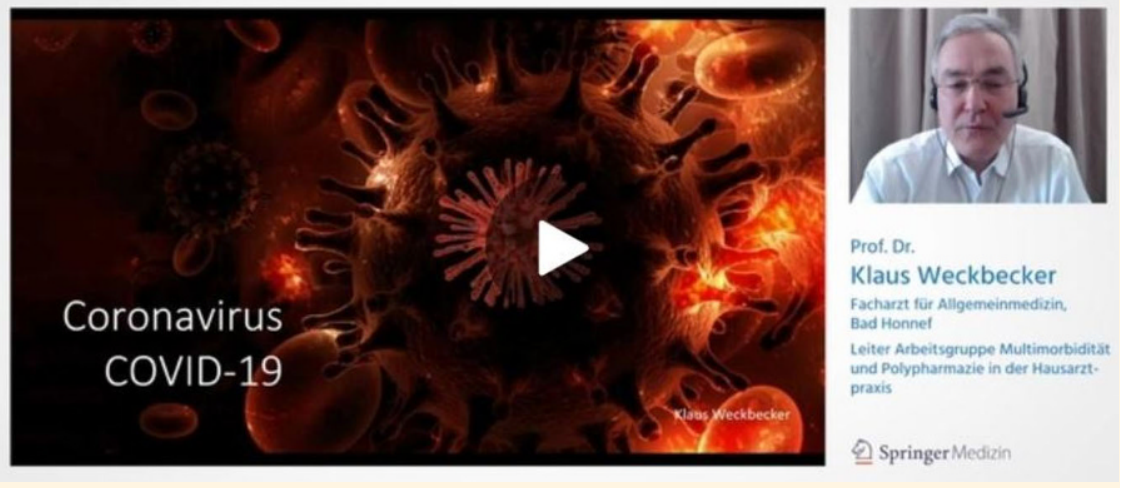

\section{Springer Medizin startet Initiative Corona-Webinar}

\section{Webinare und Videointerviews über das Ärzteportal SpringerMedizin.de}

Springer Medizin hat seit April die Initiative Corona-Webinar über sein Ärzteportal (www.springermedizin.de) gestartet. Ärzte, die auf diesem Portal registriert sind, gelangen über Videoseminare an kostenloses Wissen rund um das Corona-Virus. In jedem Webinar oder Videointerview geplant ist ein wöchentliches Erscheinen neuer Seminare - kommt ein Experte zu einem bestimmten Thema zu Wort und gibt so fachlich gesichertes Wissen an Kollegen weiter, die sich mit Symptomatik, Diagnostik und Therapie in Klinik und Praxis beschäftigen.

Auf der zentralen Corona-UpdateSeite auf SpringerMedizin.de sind unter anderem bereits folgende Webinare verfügbar:

- SARS-CoV-2-Testverfahren: Wie Sie funktionieren, was sie leisten und was nicht

- Makroskopische und mikroskopische Befunde bei COVID-19

- Infektiologe über SARS-CoV-2: "Wir werden dieses Virus nicht ewig bei uns haben"

- COVID-19-Pandemie: Aktuelles zu Epidemiologie, Diagnostik und Therapiestrategien

- Covid-19 in der Klinik: "Fast Progressors", klinische Charakteristika und prognostische Marker

- Effekt von SARS-CoV-2 auf das kardiovaskuläre System

- Covid-19 aus kardiologischer Perspektive - ein Update aus China

Alle Webinare sind über

www.springermedizin.de/covid-19 zu- gänglich, die Seite wird ständig aktualisiert und erweitert.

„In unserer Ärztekommunikation werden wir das Webinar-Format zukünftig stärker nutzen, denn es ist eine sehr wertvolle Ergänzung zu den bestehenden Print- und OnlineAngeboten", erläutert Cécile Mack, Director Digital Product \& Marketing von Springer Medizin. „Die Inhalte eines Webinars oder VideoInterviews können durch die direkte Ansprache schneller aufgenommen werden. Unsere Redakteure der verschiedenen Magazine und Zeitschriften laden zum Ausbau dieser Initiative ausgewählte Autoren und Herausgeber ein, ihr Fachwissen zu Corona zu teilen. Neben den aktuellen Aspekten werden wir auch in den nächsten Monaten das Thema eng begleiten und über die Erkenntnisse aus klinischen Studien sowie der Entwicklung von Therapieoptionen und Impfstoffen berichten. Solange die SARS-CoV-2/Covid-19-Pandemie eine Bedrohung unserer Gesellschaft darstellt, wird Springer Medizin als ein führender Marktvertreter diese Inhalte kostenfrei vor der Bezahlschranke platzieren, um allen Ärzten eine sichere und fundierte Wissensbasis zu geben. Denkbar ist außerdem, dieses Format auch auf andere medizinische Themen oder zusätzliche Fachgebiete auszudehnen", so die Digitaldirektorin. 